\title{
Cult children's toothpaste relaunches
}

Punch \& Judy, the cult children's toothpaste brand originally launched 36 years ago, has relaunched with an identity and packaging redesign retelling the classic Punch \& Judy story to appeal to modern audiences.

The new design introduces modern dad Punch who is funny, warm and prone to the occasional mishap; mum Judy who is busy, active and loves her yoga classes; and the baby, who is a fearless rebel and full of character. The crocodile, who loves to show off his gleaming smile, gets a more prominent role in the updated story.

Punch \& Judy toothpaste is already wellloved as it appeals to both children and parents. The brand comes in sugar-free: Simply Strawberry for 0-2 years old, and for 3 years and over - Hint of Mint and Fun Bubble Gum (formerly Tutti-Frutti) flavours. All flavours contain NHSrecommended levels of fluoride.

Though the Punch \& Judy story, which dates back to 1662 , has been updated, elements in the illustrations retain the story's theatrical heritage. On the front of the packs Punch, Judy and the baby are framed by theatre stage curtains.

The brand is available through the Amazon Store as of 22 June 2020.

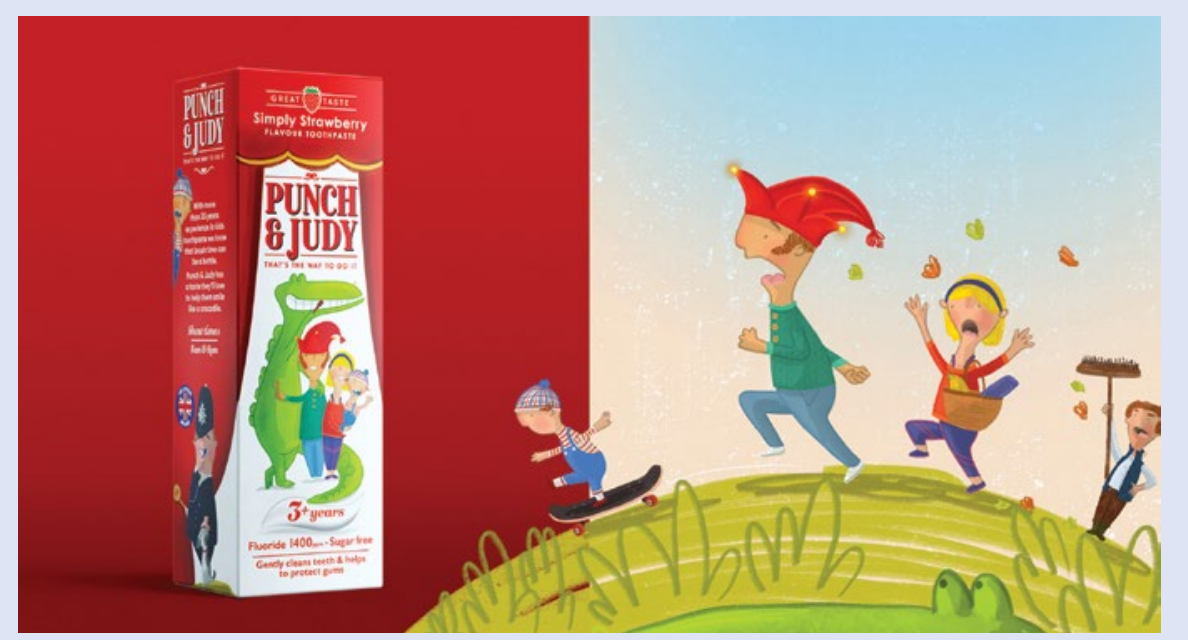

\section{New toothpaste actively strengthens children's teeth}

A new toothpaste for children aged three to six which actively strengthens and protects teeth while it cleans them has been launched in the UK and Europe. Developed by BioMin technologies, the toothpaste - BioMin F for Kids - is being released in two flavours and contains a 'smart effect' that means it is especially effective when children consume acidic foods and drinks.
BioMin F for Kids is based on the same clinically proven technology as BioMin F, helping to strengthen and protect children's teeth and preventing them from damage.

Traditional fluoride toothpastes become washed away by saliva in a relatively short time while, in contrast, BioMin's 'smart technology' controls the release of fluoride for up to 12 hours after brushing.

Professor Robert Hill worked with scientist colleagues at Queen Mary University, London to develop the toothpaste.

BioMin F for Kids contains a polymer that adheres the BioMin particles to the teeth which is slowly dissolved by saliva, continuously releasing an optimum mix of fluoride, calcium and phosphate ions to produce fluorapatite on the tooth surfaces. It is this controlled release mechanism which means that a much lower quantity of fluoride is needed to have a greater effect.

At the same time the 'smarteffect' means that in the presence of acidic food and drink in the mouth, the toothpaste starts dissolving more rapidly. This restores the mineral equilibrium and starts the remineralisation process.

Launched in two flavours, melon and strawberry, the toothpaste is available to buy directly from BioMin's distributors list on https://www.BioMin.co.uk/wherebuy. To make brushing fun and to support better oral health in children, BioMin has created 'Bino', a friendly 'binosaur' as well as developing colouring-in sheets, stickers and a useful brushing chart.

BioMin also offers two toothpastes for teenagers and adults, BioMin F and BioMin C (fluoride-free) which reduce sensitivity and strengthen and protect the tooth surfaces.

BioMin F for Kids has undergone all the appropriate cytotoxicity and biocompatibility studies ensuring its safety. It is not tested on animals and is suitable for vegans and has halal certification. The fluoride concentration at $530 \mathrm{ppm}$ is the same as the adult version, which is safe for children of three years (or those weighing at least $10 \mathrm{~kg}$ ) and above. The toothpaste is available in a silvery gel format which does not contain titanium dioxide.

www.BioMin.co.uk 